\title{
Electromagnetic Flux
}

National Cancer Institute

\section{Source}

National Cancer Institute. Electromagnetic Flux. NCI Thesaurus. Code C18941.

Changing current and concomitantly magnetic fields 